\title{
Die Ressourcensicherung von NATO und EU
}

\author{
Sind die transatlantischen Akteure Partner oder Konkurrenten im auslaufenden \\ fossilen Energiezeitalter?
}

\section{Thomas Roithner}

\begin{abstract}
The war in Iraq was symbolic for a military approach to meeting the demand of western countries for resources. A reduction of global energy policy to military instruments and alliances in global energy policy carries dangers of securitization. Among members of the EU, there is a shift from strategic planning towards concrete military missions, with the EU seeing itself as a 'global player'. A military dimension to the EU energy policy is increasingly taking shape. Co-operation between the US and the EU within NATO as an 'Energy-NATO', however, is not on the horizon. Differences and competing interests on both sides of the Atlantic are too large.
\end{abstract}

Keywords: Energiesicherheit, NATO, EU, transatlantische Beziehungen

Energy security, NATO, EU, transatlantic relations

\section{Einleitung}

Irak, Iran, das Horn von Afrika oder Afghanistan sind nur einige Beispiele von Kriegs- und Krisenschauplätzen, in denen fossile Energieträger und deren Transport in die USA oder die Europäische Union (EU) in den letzten Jahren eine zentrale Rolle spielten und die die globale Debatte um Krieg und Frieden bestimmt haben. Die NATO- und EU-Staaten stehen vor ähnlichen Problemen: steigender Energiebedarf und damit steigende Importabhängigkeit sowie alternative Energieformen mit zumeist untergeordneter Bedeutung. Die USA unter George W. Bush setzten auf konfrontative ,hard power' und die EU versucht mit ihrer Interpretation von,soft power' zum gleichen Ziel zu kommen: langfristige Verträge und Bündnisse zur Sicherung der fossilen Energiereserven viele Jahre über den ,peak oil' hinaus. Die zum Teil instabilen Ölförderregionen werden durch westliche Einflussnahme allerdings nicht stabiler. Das komplexe Spannungsverhältnis zwischen Partnerschaft und Konkurrenz von EU, USA und NATO soll in diesem Beitrag untersucht werden.

Einer der Vordenker einer alternativen Energieordnung - der Träger des Right Livelihood Award und Abgeordneter des Bundestags Hermann Scheer - zeigt sich über eine in der Zivilgesellschaft vielerorts beschworene ,mögliche andere Welt' zurückhaltend:

„Jahrhundertelang haben die europäischen Mächte ihren Kolonial-Imperialismus betrieben, um auf diesem Wege die Verfügungsgewalt über Energievorkommen und andere Rohstoffe militärisch zu sichern. Diese Kolonialzeit ist abgeschlossen und durch einen Energie-Imperialismus abgelöst worden, der für die industrialisierte Welt ökonomisch vorteilhafter ist, da sie im Normalfall auf direkte politische Unterdrückung anderer Länder verzichten und

\footnotetext{
* Mag. Dr. Thomas Roithner, Sozial- und Wirtschaftswissenschafter, Österrei chisches Studienzentrum für Frieden und Konfliktlösung (ÖSFK, Burg Schlaining), Lektor für Politikwissenschaft an der Universität Wien. Referierter Beitrag.
}

ohne administrative Verantwortung für sie auskommen kann“. 1

\section{EU-Interessen}

Die „Long Term Vision 2025“ der EU-Verteidigungsagentur geht von einer steigenden Energieabhängigkeit Europas aus. Bis 2030 wird der Bedarf um 30 Prozent ansteigen. Im Betrachtungszeitraum bis 2025 wird die externe Abhängigkeit von Öl auf 90 Prozent und die von Gas auf 80 Prozent ansteigen. Immer wieder melden sich Kritiker - wie US-Senator Richard Lugar - zu Wort, die die EU vor zu starker Abhängigkeit von Russland warnen. ${ }^{2}$ China und Indien fördern fossile Energieträger in Asien, Afrika und dem Nahen Osten - oftmals unter Bedingungen, denen die Menschenrechtskonditionalität der EU offizielle Grenzen setzt - und die Suche der EU-Staaten nach Energie wird sich nicht nur auf die europäische Nachbarschaft beschränken lassen. ${ }^{3}$ Die Energiefrage wird, bei einer Beibehaltung des auf Öl und Gas setzenden Systems, angesichts des steigenden Bedarfs zu einem zentralen Legitimationsgrund für offensiven Interventionismus werden. „Was im Cheney-Bericht explizit ausgeführt wurde, bestimmt auch die Energiepolitik der EU: Rekurs auf die Regeln und Wohltaten des freien Handels (...) und schließlich politischer und militärischer Druck, wenn die anderen Mittel versagen". 4

In der Öffentlichkeit der EU-Staaten hat man durch die 2006 ausgelöste Debatte um die Energiesicherheit den Versuch ge-

1 Hermann Scheer, Sonnenstrategie. Politik ohne Alternativen, München, 1993, S. 34.

2 Ida Garibaldi, NATO and European Energy Security, American Enterprise Institute for Public Policy Research, No. 1, Washington D.C., 2008; Paul Gallis, NATO and Energy Security, Congressional Research Service, Update, Washington D.C., 15.8.2007, S. 2.

3 European Defence Agency (EDA), An Initial Long-Term Vision für European Defence Capabilitiy and Capacity Needs, endorsed on $3^{\text {rd }}$ October 2006, Brussels, point 11.

4 Elmar Altvater, Sicherheitsdiskurse beiderseits des Atlantik - In Zeiten von Peak Oil und Klimawandel, in: ÖSFK / Thomas Roithner (Hg.): Von kalten Energiestrategien zu heißen Rohstoffkriegen?, Münster/Wien, 2008, S. 44-71, hier: S. 54. 
macht, Rohstoffsicherungspolitik unterschiedlicher Intensität - und hier sei nicht nur an militärische Aspekte gedacht - in die Diskussion zu bringen, um sie als offizielle Doktrin der EU festzuschreiben. Flankiert von Teilen der nationalen politischen und militärischen Eliten hat die Europäische Sicherheitsstrategie (ESS) ${ }^{5}$ einen offiziellen Schritt der EU in diese Richtung bereits unternommen. Die Energieabhängigkeit ist ein „Anlass zur Besorgnis“. Der Hohe Vertreter für die Außen- und Sicherheitspolitik der EU ruft zum „gemeinsamen Handeln“ auf und legt dar: „Schließlich müssen wir unser Engagement im Nahen Osten ausbauen. Dieser wird auf Jahre die Hauptquelle für Europas Energie bleiben“. ${ }^{6}$ Vor diesem Hintergrund ist es nicht verwunderlich, wenn der deutsche Energiestaatssekretär Joachim Wuermeling meint: „Im globalen Kampf um Energiequellen muss die EU härtere Bandagen anlegen“. ${ }^{7}$

Im Jahr 2004 hat der EU-Rat das „European Defence Paper“ (EDP) in Auftrag gegeben. Man geht von der „Transformation Europäischer Streitkräfte von der Landesverteidigung in Richtung Intervention und Expeditionskriegszüge"8 aus. Ein künftiges militärisches Szenario könnte wie folgt aussehen:

„In einem Staat X am Indischen Ozean haben antiwestliche Elemente die Macht erlangt und benützen das Öl als Waffe, vertreiben westliche Bürger und greifen westliche Interessen an. Darüber hinaus haben sie mit der Invasion des Nachbarlandes Y begonnen, dessen Regime pro-westlich orientiert ist und eine zentrale Rolle beim freien Fluss von Öl in den Westen spielt. [...] Die EU interveniert gemeinsam mit den USA und mit einer starken Streitmacht, um das Land Y zu unterstützten und ihre eigenen Interessen zu schützen. [...] Das militärische Ziel der Operation ist es, das besetzte Territorium zu befreien und Kontrolle über einige der Öl-Infrastrukturen, Pipelines und Häfen des Landes X zu bekommen." 9

Die EU brauche für derartige Aufgaben eine entsprechende „militärische Eskalationsdominanz" ${ }^{10}$ Vor allem Afrika dient künftig als Testgelände für die ,EU Battlegroups'.

Bei dem im Dezember 2008 vorgelegten EU-Bericht zur Umsetzung der ESS spielt die Frage der Energiesicherheit eine bedeutendere Rolle als noch 2003. Man spricht sich für „eine stärkere Diversifizierung der Brennstoffe, Versorgungsquellen und Transitrouten " aus. ${ }^{11}$ Bei der Förderung alternativer Energieträger scheinen die EU-Mitglieder hingegen mit der Formel „man sollte erneuerbare Energie fördern“ einen großen Schritt rückwärts zu gehen.

5 Europäische Union, Ein sicheres Europa in einer besseren Welt. Europäische Sicherheitsstrategie, Brüssel, 12.12.2003.

6 Javier Solana: Gemeinsam handeln, in: Financial Times Deutschland, 9.3.2006.

7 Bundesministerium für Wirtschaft und Technologie, Pressemitteilung vom 22.9.2006, Energiestaatssekretär Dr. Joachim Wuermeling: http://www.bmwi. de/BMWi/Navigation/Presse/pressemitteilungen,did=158972.html.

8 Institute for Security Studies (ISS), European Defence - A Proposal for a White Paper, Paris, 2004, S. 55: http://www.iss.europa.eu/uploads/media/wp2004. pdf.

9 ISS, a.a.O., S. $83 \mathrm{f}$

10 ISS, a.a.O., S. 105.

11 Europäischer Rat, Bericht über die Umsetzung der Europäischen Sicherheitsstrategie - Sicherheit schaffen in einer Welt im Wandel, Bericht des Generalsekretärs/Hohen Vertreters für den Ausschuss der Ständigen Vertreter/Rat, 16823/1/08, REV 1, Brüssel, 5.12.2008.
Unübersehbar ist auch das Streben der EU nach Einfluss in Zentralasien. In der ,Strategie für eine neue Partnerschaft' mit Zentralasien, ${ }^{12}$ die unter deutscher EU-Ratspräsidentschaft beschlossen wurde, kommt das Wort ,Energie' 52-mal vor - womit die EU ihre Prioritäten klar festlegt. Die Frankfurter Allgemeine Zeitung verweist auf eine Milliarde Euro, die in den letzten 15 Jahren in die Region Zentralasien geflossen sind. Dies sei „keine große Investition, zumal die EU wirtschaftlich mit Russland und China um die Gunst Zentralasiens konkurriert“.13 Die unterkühlte Stimmung des jüngsten EU-Russland-Gipfels in Chabarowsk im Mai 2009 war eine Folge der neuen Zentralasien-Kooperation der EU. ${ }^{14}$

Die Armeen der EU-Mitgliedstaaten haben - Scheers pessimistische Prognose aus dem Jahr 1993 bestätigend - den Zugang zu Rohstoffen auf dem Papier längst zur militärischen Aufgabe erklärt. Beispielsweise schreiben die Verteidigungspolitischen Richtlinien der Deutschen Bundeswehr von 1992 fest, dass es um die „Aufrechterhaltung des freien Welthandels und des ungehinderten Zugangs zu Märkten und Rohstoffen in aller Welt im Rahmen einer gerechten Weltwirtschaftsordnung “15 gehen müsse, während es die Richtlinien 2003 etwas dezenter formulieren. Das 2006 in Deutschland erschienene Weißbuch befürchtet „Störungen der Rohstoff- und Warenströme, beispielsweise durch zunehmende Piraterie“. ${ }^{16}$ Deutschland bleibt mit der Formulierung rohstoffinterventionistischer Gedanken innerhalb der EU-Staaten kein Einzelfall, wenngleich die operative Ebene mit den Strategiepapieren oft nicht Schritt hält. Besonders auf die zahlreichen Beispiele der Interventionspolitik Großbritanniens und Frankreichs sei hier hingewiesen. In der österreichischen Sicherheitsdoktrin aus dem Jahr $2001^{17}$ erklärt man die „Sicherstellung lebensnotwendiger Ressourcen“ zu einer der wichtigsten politisch-strategischen Zielsetzungen Österreichs. Die Website des Verteidigungsministeriums bricht die wichtigste Aufgabe des Heeres - Auslandseinsätze im Rahmen der EU - auf die Ressourcensicherung herunter und führt aus:

„Für Österreich ist die Teilnahme an EU-Einsätzen mit großem Nutzen verbunden (...). Als wesentliche Zielsetzung der europäischen Sicherheitspolitik nennt Prof. DDr. Erich Reiter, Beauftragter für Strategische Studien des BMLV: (...) Kooperation mit den USA und mit Japan zum globalen Management von Konflikten und zwecks Zugang zu strategischen Rohstoffen, der Aufrechterhaltung des freien Handels und der Schiffahrt". ${ }^{18}$

Sowohl Militär- als auch Energiepolitik sind nach wie vor faktisch stark nationalstaatlich geprägte Politikbereiche, was jedoch bislang ein Zusammendenken und eine Weiterent-

12 Europäische Union, Die EU und Zentralasien: Strategie für eine neue Partnerschaft: http://www.auswaertiges-amt.de/diplo/de/Europa/Aussenpolitik/ Regionalabkommen/Zentralasien-Strategie-Text-D.pdf.

13 Wulf Schmeise, Die „Tankstelle Europas“ anzapfen. Steinmeier in Zentralasien, in: Frankfurter Allgemeine Zeitung, 28.3.2007..

14 Verena Diethelm, Moskau will Brüssel seine Größe zeigen, in: Der Standard, 22.5.2009.

15 Bundesminister der Verteidigung, Verteidigungspolitische Richtlinien der deutschen Bundeswehr, Bonn 26.11.1992, Kapitel II, Punkt 8 (8).

16 Bundesministerium der Verteidigung, Weißbuch 2006 zur Sicherheitspolitik Deutschlands und zur Zukunft der Bundeswehr, Berlin, 2006, S. 22.

17 Bundesministerium für Landesverteidigung, Sicherheits- und Verteidigungsdoktrin, Analyse-Teil, Wien, 2001.

18 Bundesministerium für Landesverteidigung, „Sicherheit mit moderner Technik“ (Web-Dokument): http://www.bmlv.gv.at/cms/artikel.php?ID=1783. 
wicklung dieser Bereiche nicht gänzlich behindert. Über gemeinsame außen-, sicherheits- und verteidigungspolitische Initiativen westeuropäischer Staaten denkt man bereits seit der zweiten Hälfte der 1940er Jahre intensiv nach. Der Beschluss zum Aufbau einer integrierten EU-Energiepolitik fiel beim EURat im März 2007.

\section{US-Interessen}

Ähnlich der Daten der EU-Verteidigungsagentur hat die National Energy Policy Group in den Vereinigten Staaten bereits vor den Terroranschlägen des 11. September 2001 Prognosen zur Energiesicherheit für die nächsten 25 Jahre - das Papier wurde unter dem Titel ,Cheney-Report' bekannt - vorgelegt. Das Dokument ist in engem Zusammenhang mit der Nationalen Sicherheitsstrategie (NSS) der USA zu sehen und legt dar, dass der Anteil des importierten Öls im Jahr 2020 von 52 auf 66 Prozent ansteigen wird. Gewarnt wird vor der Energieabhängigkeit von einer einzigen Weltregion. Eine Diversifizierung ist daher ein logischer Schritt. ${ }^{19}$ Schon 1980 tönte der heute als ,Taube' firmierende demokratische US-Präsident Jimmy Carter: „Jeder Versuch einer auswärtigen Macht, die Kontrolle über den Persischen Golf zu erlangen, wird als Angriff auf die vitalen Interessen der USA betrachtet und (...) mit allen erforderlichen Mitteln, einschließlich militärischer, zurückgeschlagen werden “ ${ }^{20}$ Für die USA ging es unter Bill Clinton u.a. um lebenswichtige Interessen, wozu auch „die Sicherung uneingeschränkten Zugangs zu Schlüsselmärkten, Energievorräten und strategischen Ressourcen“21 gehörte. Dreieinhalb Jahre nach dem George W. Bush den Irak-Krieg als ,Mission Accomplished' bezeichnet hatte, führte Jeffrey D. Sachs - Wirtschaftswissenschafter der Columbia University - aus:

„Seit Winston Churchill zu Beginn des 20. Jahrhunderts die Umrüstung der britischen Marine von Kohle auf Ö1 einleitete, haben sich die westlichen Mächte unaufhörlich in die Angelegenheiten nahöstlicher Länder eingemischt, damit das Öl weiterfließt. Doch das Spiel ist so gut wie vorbei, denn die alten Methoden versagen offensichtlich. “22

Vor dem Hintergrund des steigenden Energiebedarfs hat auch Afrika für die USA wieder geostrategische Bedeutung erlangt und es wurde ein eigenes Afrika-Kommando (AFRICOM) eingerichtet. Afrika verfügt über rund zehn Prozent der globalen Öl- und acht Prozent der Gasvorräte, wobei auf dem Kontinent noch große, bislang unentdeckte Lagerstätten vermutet werden. ${ }^{23}$ Neben den Schauplätzen im Nahen und Mittleren Osten, in Afrika und Lateinamerika ortet Elmar Altvater -

19 Vgl. hierzu: http://www.whitehouse.gov/infocus/energy/.

20 Zit. nach Otfried Nassauer: Der Ruf nach Energiesicherheit. Herausforderungen für eine neue Sicherheitspolitik, NDR-Info, 10. März 2007.

21 Bill Clinton vor den Vereinten Nationen am 27.9.1993, zit. nach Noam Chomsky, War against People. Menschenrecht und Schurkenstaaten, Hamburg, 2001, S. 12. Was die bereits in die 1920er Jahre zurückreichenden Begehrlichkeiten betrifft, so sei hier verwiesen auf Karin Kneissl, Die Geopolitik des Erdöls und Erdgases: Kriegerischer oder friedlicher Zugang zu den Reserven am Golf, in: ÖSFK (Hg.): Krisenherd Naher und Mittlerer Osten, Münster/Wien, 2007, S. 152-169, hier S. 154.

22 Jeffrey D. Sachs, Alternative zur Zukunft Bushs, in: Der Standard, 7./8.10.2006.

23 Jürgen Wagner, Das neue Objekt der Begierde: Afrikanische Ölkriege und die Rolle des „Westens“, IMI-Studie 2007/09, Tübingen, S. 2 f.
Politologe an der Freien Universität Berlin - in Zentralasien und dem Kaukasus ein „Pipelineistan“. ${ }^{24}$

Die engagierten Pläne der Administration Obama ${ }^{25}$ - wenngleich die meisten Überprüfungszeiträume weit jenseits von zwei Amtsperioden liegen - sehen enorme Sparpotenziale für Öl vor und streben eine Reduktion der Treibhausgasemissionen um 80 Prozent bis 2050 an.

\section{Auf dem Weg zur ,Energie-NATO'?}

Auf dem NATO-Gipfel in Rom 1991 wurde ein neues strategisches Konzept verabschiedet. ${ }^{26}$ Risiken seien „schwerer vorauszusagen“ (Ziffer 9) und Bedrohungen könnten sich aus „der Unterbrechung der Zufuhr lebenswichtiger Ressourcen“ sowie aus „Terror- und Sabotageakten“ (Ziffer 13) ergeben. Das Einsatzgebiet der NATO wurde über das Bündnisgebiet hinaus ausgedehnt (Out of area-Einsätze). Die Sicherheitsinteressen der NATO können gemäß ihres Strategischen Konzepts von $1999^{27}$ von der „Unterbrechung der Zufuhr lebenswichtiger Ressourcen“ (Ziffer 24) berührt werden. Gerade die Legitimationskrise der NATO - Stichwort Afghanistan, Erweiterung, Raketenabwehr - hat einige Beobachter und Beobachterinnen veranlasst, die Energiesicherheit als eine zentrale Aufgabe der Zukunft für das Bündnis zu betrachten. ${ }^{28}$ Der US-amerikanische Kongressabgeordnete Tom Lantos verdeutlichte in der Zeitschrift NATO Review $^{29}$ die Bedeutung der Energiefrage für ein umfassendes Sicherheitsverständnis und die Geopolitik. Sie sollte daher auch für die NATO Vorrang haben. Dies begründete Lantos zusätzlich mit der aus seiner Sicht aggressiven Energiepolitik Russlands.

Von den aktuell laufenden NATO-Missionen weisen beinahe alle einen Bezug zur Rohstoffsicherung auf: ${ }^{30}$

NATO Counter-Piracy am Horn von Afrika

seit 03/2009

NATO Assistance der AU (Somalia, Sudan)

seit 2005 und 2007

NATO Assistance in Irak

seit $07 / 2004$

ISAF in Afghanistan

seit $08 / 2003$

Operation Active Endeavour im Mittelmeer

seit $10 / 2001$

Kosovo Force (KFOR) im Kosovo

seit 06/1999

Trainings und Übungen - wie beispielsweise ,Steadfast Jaguar' der NATO Response Force (NRF) auf den Kapverden - finden u.a. mit dem Ziel der Sicherung energetischer Ressourcen statt.

24 Elmar Altvater, Geopolitische Konflikte am Ende des fossilen Energieregimes, in: ÖSFK / Thomas Roithner (Hg.): Die Weltunordnung von Ökonomie und Krieg, 3. Aufl., Münster/Wien, 2006, S. 58-81, hier: 76.

25 Barack Obama, Joe Biden, New Energy for America: http://www.barackobama. com/pdf/factsheet_energy_speech_080308.pdf.

26 NATO, The Alliance's New Strategic Concept, Brussels, 7. -8.11. 1991.

27 NATO, The Alliance's Strategic Concept, Brussels, 23.-24.4.1999.

28 Beispielsweise Michael Rühle, Der steinige Weg ins globale Zeitalter, in: Internationale Politik, 3/2008, 63. Jg., Frankfurt/Main, S. 6-15, hier: 14.

29 Tom Lantos: Energy security: A state side view, Interview, NATO review, issue 4/2007: http://www.nato.int/docu/review/2007/issue4/english/interview2. html.

30 Aufstellung aus: http://www.nato.int/cps/en/natolive/topics_52060.htm. 
Der ehemalige NATO-Generalsekretär Jaap de Hoop Scheffer sprach auf der Münchner Sicherheitskonferenz $2008^{31}$ von einer Herausforderung durch die „Verwundbarkeit unserer Energievorsorgungsleitungen“. Vor einem Publikum, das einer stärkeren Rolle der NATO sicherlich nicht abgeneigt ist, führte de Hoop Scheffer aus: „Die Schlüsselfrage ist: Wie können wir all die aufkommenden neuen Wirtschaftsgiganten überzeugen, dass Energie allen gehört und sie mit uns im Management dieser knappen Ressource kooperieren. “32 Er kommt zu dem Schluss, dass steigende Preise und damit steigende Einnahmen für ölproduzierende Staaten auch größeren Einfluss bei der Gestaltung der globalen Sicherheitsordnung zur Folge haben werden.

Die NATO verfügt über mehrere bereits angewandte bzw. in intensiver Debatte befindliche Möglichkeiten zur Sicherung von Ressourcen, darunter etwa:

- Polizeifunktion zur Sicherstellung einer reibungslosen Schifffahrt,

- Partnerschaften, Dialog und Informationsaustausch,

- ,Security Assistance' sowie

- ,Interdiction Operations'.

\subsection{Polizeifunktion}

Die NATO kann nach Ansicht von Jaap de Hoop Scheffer eine Polizeifunktion einnehmen und die reibungslose Schifffahrt auf hoher See schützen. Marineeinheiten fungieren immer öfter als Schnittstellen zwischen nationaler und internationaler Sicherheit. Seit 2001 ist die NATO mit der ,Operation Active Endevour' neben der Terrorabwehr ,auch indirekt eine Unterstützung, um Versicherungsprämien für die kommerzielle Schifffahrt niedrig zu halten“. Derzeit kooperiert die NATO mit der EU, um Piraterie, die sich gegen die kommerzielle Schifffahrt vor Somalia richtet, zu stoppen.

Allerdings, so räumt de Hoop Scheffer auf Anfrage großer Ölkonzerne ein, gibt es durchaus Probleme bei der militärischen Eskortierung privater Tankerflotten. Dies betrifft insbesondere die Kosten der Einsätze. Die NATO sei aber selbstverständlich bereit, einen Dialog mit den Ölkonzernen zu beginnen, um „Öl und Gas so sicher und billig wie möglich zu uns nach Hause und zu unseren Industrien zu bringen“. .3

\subsection{Partnerschaften, Dialog und Information}

Neben der Polizeifunktion zum Schutz der Öl- und Gasversorgung sieht die NATO ihre Aufgabe im Auf- und Ausbau von Partnerschaften. Diese sind nicht nur mit Förderländern vorgesehen, sondern erstrecken sich auch auf die Transitstaaten. Wenngleich andere internationale Organisationen nach An-

31 Jaap de Hoop Scheffer, Rede anlässlich der Münchner Sicherheitskonferenz 2008, in: Europäische Sicherheit, Nr. 3/2008, 57. Jg., Bonn, S. 24-26, hier: S. 24.

32 Jaap de Hoop Scheffer, "Energy security in the $21^{\text {st }}$ century", Keynote speech by NATO SG at the Economist Energy Security dinner, Lloyd's, London, 23.10.2008

33 Jaap de Hoop Scheffer, Keynote speech. sicht des ehemaligen NATO-Generalsekretärs bessere Möglichkeiten für diesbezügliche Aktivitäten besitzen, so sollten sich die Verbündeten dennoch um eine gemeinsame Analyse des strategischen Werts von Energiesicherheit bemühen, um gemeinsame Ansätze zu entwickeln.

Der NATO-Gipfel in Riga am 29. November $2006^{34}$ legte in Fragen der Energiesicherheit den Arbeitsauftrag offen, „die Bereiche zu definieren, in denen die NATO zur Gewährleistung der Sicherheitsinteressen ihrer Mitglieder einen Mehrwert einbringen und auf entsprechendes Ersuchen nationale und internationale Anstrengungen unterstützen kann“ (Punkt 45). Am 18. November 2008 wurde im spanischen Valencia eine NATOResolution $^{35}$ verabschiedet, auf deren Grundlage bei der NATO ein „Center of excellence“ eingerichtet werden soll, „um energiebezogene Bedrohungen zu identifizieren und zu analysieren und um Ratschläge anzubieten, wie man diese überwinden kann“. Der Politikwissenschaftler Johannes Varwick schlägt in diesem Zusammenhang vor, dass die NATO zur Erhöhung der Energiesicherheit die nationalen Geheimdienste koordinieren könnte oder diese Aspekte in der NATO-PfP - die auch die zentralasiatischen Staaten umfasst - verankert. ${ }^{36}$ Dies wäre seiner Ansicht ein Mehrwert der NATO, ohne dass Aufgaben anderer internationaler Institutionen dupliziert werden würden.

Beim NATO-Gipfel in Straßburg und Kehl im April 2009 wurde dargelegt, dass die „politische Absprache und die praktische Kooperation intensiviert wurden und neue Möglichkeiten in Schlüsselbereichen wie Energiesicherheit, maritime Sicherheit, Training und Ausbildung geschaffen wurden“. ${ }^{37}$ Dabei spielen angesichts der Probleme einiger NATO-Staaten mit der Gasversorgung im Januar 2009 Fragen von stabiler und verlässlicher Lieferung sowie der Diversifizierung der Transportrouten, Anbieter und Energieträger eine wichtige Rolle.

\section{3 „Security Assistance“}

Unter der von Jamie Shea ${ }^{38}$ - heute Direktor für Policy Planning im Private Office des NATO-Generalsekretärs in Brüssel - benannten Aufgabe der „Security Assistance“ können sicherlich in Teilen auch die beim NATO-Rat in Bukarest am 3. April 2008 im Bereich Energiesicherheit (Punkt 48) ${ }^{39}$ offiziell benannten Aufgaben des ,projecting stability' und der ,protection of critical energy infrastructure' verstanden werden.

34 NATO, Riga Summit Declaration. Issued by the Heads of State and Government participating in the meeting of the North Atlantic Council in Riga on 29 November 2006: http://www.nato.int/cps/en/natolive/official_texts_37920. htm.

35 NATO Parliamentary Assembly, Resolution 372 on Energy and Security, Brussels, 18.11.2008

36 Johannes Varwick, Die militärische Sicherung von Energie, in: Internationale Politik, Nr. 3/2008, 63. Jg., Frankfurt/Main, S. 50-55, hier: S. 51 ff.

37 NATO, Strasbourg / Kehl Summit Declaration. Issued by the Heads of State and Government participating in the meeting of the North Atlantic Council in Strasbourg / Kehl, 4.4.2009, point 38: http://www.nato.int/cps/en/natolive/ news_52837.htm.

38 Jamie Shea, Energy security: NATO's potential role, in: NATO review, Autumn 2006.

39 NATO, Bucharest Summit Declaration. Issued by the Heads of State and Government participating in the meeting of the North Atlantic Council in Bucharest on 3 April 2008: http://www.nato.int/cps/en/natolive/official_texts 8443.htm. 
Die von Shea - seinerzeit Leiter des NATO-Referats für politische Planung - vorgeschlagene flexibel funktionierende „Security Assistance“ „könnte unter extremen Umständen energetische Besitztümer auch durch die Aktivierung von schnellen Reaktionskräften - die ständig in Bereitschaft auf stand-by gehalten werden könnten - beschützen“. Zudem schlägt Shea angesichts der ,Operation Active Endeavour' eine „maritime Überwachung und bedrohungsbezogene Antwort “40 vor. Da die NATO im Zuge dieser Maßnahmen nicht alle Seewege überwachen könne, plädiert Shea für einen Schutz von kritischen Nadelöhren (choke points), je nach Konfliktlage. Auf diese Weise könnte die Marine rascher an diesen Punkten eingreifen. Kritiker befürchten allerdings, die NATO könne so zu einer „Eskorte von Öl- und Gaskonzernen“ mutieren. ${ }^{41}$

\section{4 „Interdiction Operations“}

Als weitere Variante bringt Shea die „Interdiction Operations“ ins Gespräch. Dabei handelt es sich um „Militärinterventionen, die explizit entwickelt werden, um die Lieferung von Öl oder Gas aus aktuellen Krisen- oder Konfliktsituationen zu sichern “. ${ }^{42}$ Pate dieses Gedankens ist die ,Operation Earnest Will” von 1987 bis 1988, die den Schutz kuwaitischer Öltanker im Iran-Irak-Krieg gewährleistet hat. Solche Maßnahmen können auch den Schutz von Ölterminals, den militärischen Schutz nationaler Einrichtungen beim Be- und Entladen sowie den Schutz von Raffinerien und Lagerstätten umfassen. Nach Shea verlangen diese Militärinterventionen eine eigene Planung, eigene multinationale Übungsszenarien für die Marine und andere involvierte Militäreinheiten in der Luft und am Boden.

Während sich die Politik der NATO-Staaten in Bezug auf die Rohstoffsicherung militarisiert, muss festgestellt werden, dass die Erforschung alternativer Energieträger und die Dezentralisierung des Energiesystems in den Dokumenten der NATO nur eine äußerst untergeordnete Rolle spielt. Das Ausblenden von Konfliktursachen und Alternativen - die NATO sucht in zahlreichen, klassisch zivilen Bereichen mit den ihr zur Verfügung stehenden Mitteln nach Legitimation und Aufgaben - trägt zu einer gefährlichen ,Versicherheitlichung' und Militarisierung der internationalen Beziehungen bei.

\section{Transatlantische Rohstoffagenda - transatlan- tischer Dissens}

Die NATO-Staaten wurden bis zum Fall der Berliner Mauer durch den gemeinsamen Feind im Osten zusammengehalten. Seither ist man in Brüssel und Washington in unterschiedlicher Intensität auf der Suche nach einer neuen gemeinsamen Klammer, wobei sich weder der Terrorismus noch Massenvernichtungswaffen oder Huntingtons, Clash of Civilizations' als ein nachhaltig neues und einheitliches Feindbild des Westens etablieren konnten. Gemeinsamer militärischer Interventionismus hat unter George W. Bush für einige europäische Verbündete

40 Jamie Shea, a.a.O

41 Hermann Scheer, Kein friedliches Europa ohne solare Revolution, in: ÖSFK / Thomas Roithner (Hg.), a.a.O., S. 133-146, hier: S. 143.

42 Jamie Shea, a.a.O. deutlich an Attraktivität verloren. Vielmehr haben zentrifugal wirkende Ereignisse wie der Irak-Krieg, der Dissens um den internationalen Strafgerichtshof, die Terrorbekämpfung à $l a$ Guantanamo oder die US-Raketenabwehr zu einem sichtbaren Riss in den transatlantischen Beziehungen geführt.

Mindestens drei Gründe lassen Zweifel aufkommen, dass die NATO mit ihren heute vorhandenen Instrumenten gegenwärtig geeignet und in der Lage ist, die Aufgabe der Rohstoffsicherung zu übernehmen:

- Erstens brauchen die USA die NATO aus militärischer Sicht nicht. Wenn die nationale Energiesicherheit der USA ernsthaft tangiert ist, wird sich das Handeln Barack Obamas nicht wesentlich von dem seiner Vorgänger unterscheiden. Militärische Einsätze der USA, denen auch nur der Geruch von Rohstoffinterventionismus vorausweht, würden im Rahmen der UNO vermutlich ein Veto von Seiten der Russischen Föderation und China zur Folge haben. Auch einzelne europäische Verbündete könnten - trotz der deutlich sichtbaren Relativierung eines nötigen UN-Sicherheitsratsmandates für Interventionen im Rahmen der EU - Bedenken unterschiedlichster Art vorbringen.

- Zweitens hat die EU - allen voran Deutschland und Frankreich - 2007 ihre ,battle groups' für einsatzbereit erklärt und ist fähig und willens, autonome Militäreinsätze im gesamten Petersberger Aufgabenspektrum abzudecken. Beispielsweise hat das Georgien-Mandat der EU u. a. die Beobachtung der Energie-Infrastruktur und der entsprechenden Versorgungseinrichtungen ${ }^{43}$ zum Inhalt. Statt im Namen der NATO, im Dienste der USA und auf Kosten der EU zu potenziellen Militäreinsätzen zu schreiten, scheint die Weiterentwicklung eigener Interventionskapazitäten aus Sicht der politischen und militärischen EUEliten wohl sinnvoller. Einige EU-Staaten wie Großbritannien oder Polen orientieren sich in diesen wie auch anderen Fragen allerdings eher an den USA und der NATO. Auch der verbale Einstieg der EU in den Ressourcenwettlauf in der Arktis ${ }^{44}-\mathrm{u}$. a. in Konkurrenz zu den USA, Russland oder Kanada - deutet auf eine wachsende Rolle der EU als ,global player' hin.

- Drittens ist es für China, Indien, Russland, Iran, Venezuela u. a. - gleichgültig, ob Ressourcenimporteure oder -exporteure nicht unwesentlich, ob die führenden westlichen Militärmächte gemeinsam oder getrennt nach den Ressourcen im Nahen und Mittleren Osten, in Zentralasien, Afrika und Lateinamerika zu greifen versuchen. Einzelne Nationalstaaten und Staatenkooperationen wie die SCO (Shanghai Cooperation Organization, der Russland, China, Kasachstan, Kirgistan, Tadschikistan und Usbekistan angehören), die BRIC (Brasilien, Russland, Indien, China), die UNASUR (Union Südamerikanischer Nationen) oder die Arabische Liga lehnen ein gemeinsames Vorgehen ab. Auch die Proteste der Zivilgesellschaft angesichts des Jubiläumsgipfels der NATO haben gezeigt, dass Teile der Öffentlichkeit nicht nur die Auslandseinsatzpolitik der NATO, sondern auch deren Verschlossenheit gegenüber Alternativen kritisch bewerten.

43 European Union, Council Joint Action on the European Union Monitoring Mission in Georgia, EUMM Georgia, 2008/736/CFSP, Brussels,15.9.2008, Article 3.2: http://eur-lex.europa.eu/LexUriServ/LexUriServ.do?uri=OJ: L:2008:248:0026:0031:EN:PDF.

44 Wolfgang Tucek, EU liebäugelt mit Arktis-Ressourcen. Schwieriger Wettlauf mit Russland steht bevor, in: Wiener Zeitung, 26.11.2008. 
Die geopolitischen Interessen der transatlantischen Akteure USA und EU zur Ressourcensicherung sind heute zu konkurrierend und gegensätzlich, als dass sie unter dem Dach der NATO vereinigt werden könnten. In der Frage der militärischen, diplomatischen und ökonomischen Ressourcensicherung werden die transatlantischen Spannungen in den folgenden Jahren eher zu- als abnehmen, auch wenn die USA gegenwärtig an einer Stärkung und Aufwertung der NATO interessiert sind. Nicht auszuschließen ist allerdings, dass der Druck der russischen, chinesischen oder indischen Politik die USA und die EU sicherheitspolitisch wieder zusammenführen - allerdings kaum unter dem Dach der heute existierenden NATO.

\section{Alternativen jenseits westlicher Interventionen}

Wir müssen heute feststellen, dass Konflikte um Ressourcen auch im Kleid der Sicherung der Menschenrechte, Abrüstungskriege, des ,war on terror', der Militärpräsenz im Sinne der Sicherung einer ,good governance' oder künftig vielleicht im Gewand der ,responsibility to protect' auf uns zukommen.
Hermann Scheer betont den „weit reichendsten wirtschaftlichen Strukturwandel seit Beginn der industriellen Revolution“,45 der durch erneuerbare Energieträger ausgelöst werden kann. Auf den einleitend von ihm skizzierten „Energie-Imperialismus" kann heute eine weitere Fortschreibung der Besitzverhältnisse in Bezug auf alternative Energieformen befürchtet werden. Wenn Öl, Gas oder Uran nun durch Sonne, Wind und Wasser ersetzt werden, sich aber an den Besitzverhältnissen bei Kraftwerken, Leitsystemen oder der Distribution nichts ändert, wird die erwartete „Revolution“ ausbleiben. Eine demokratische und dezentral organisierte Substituierung der Energieträger ist nicht nur eine ökologische, sondern auch eine verteilungspolitische und friedenspolitische Frage. Die damit im Zusammenhang stehenden globalen Herausforderungen sind nichtmilitärischer Natur und verlangen nichtmilitärische und multilaterale Ansätze zu ihrer Bearbeitung. Weder die NATO Response Force noch die ,EU battle groups' können diese Probleme lösen.

45 Hermann Scheer, Energieautonomie. Eine neue Politik für erneuerbare Energien, München, S. 13.

\title{
Die geopolitische Dimension der Ressourcensicherheit - eine wachsende Herausforderung für Deutschland und Europa
}

\author{
Heinrich $\mathrm{Kreft}^{*}$
}

\begin{abstract}
Mineral raw materials are a key factor for sustainable growth in Germany and the EU. Unprecedented demand in particular from China has affected an increase in prices - a trend which is likely to continue. Germany and the EU are highly dependent on imports, including many high-tech minerals. Concerns that arise are thus the stability of producer countries, free access to raw materials and how to assist producer countries in managing resource extraction in a sustainable manner and to the benefit of their citizens. Resource security is a responsibility of private companies. But in order to address the growing political issues, an integrated approach is needed at the national, European and international level, which involves diplomacy, trade and development, as well as competitiveness, environmental and social policies.
\end{abstract}

Keywords: Rohstoffsicherheit, Importabhängigkeit, Hochtechnologiemetalle, Rohstoffdiplomatie, nachhaltiges Ressourcenmanagement

Security of resources, dependency on imports, high-tech metals, resource diplomacy, sustainable resource management

\section{Einleitung}

Rohstoffe sind für moderne Industrie- und Dienstleistungsgesellschaften unverzichtbar. Daher ist eine sichere Rohstoffversorgung zu akzeptablen Preisen von existenzieller Bedeutung für Deutschland und Europa.

\footnotetext{
* Dr. Heinrich Kreft ist Karrierediplomat mit Tätigkeiten an der Deutschen Botschaft in Bolivien, Japan und den USA. Zuletzt war er stellvertretender Leiter des Planungsstabs im Auswärtigen Amt. Derzeit ist er außen- und sicherheitspolitischer Berater der CDU/CSU-Bundestagsfraktion in Berlin.
}

Steigende Energiepreise und der wiederholte Gaskonflikt zwischen Russland und der Ukraine haben die Abhängigkeit Deutschlands und Europas von Öl- und Gasimporten für alle deutlich gemacht und auf die politische Agenda gesetzt. ${ }^{1}$ Vergleichbare Probleme bei der Versorgung mit nicht-energetischen Rohstoffen haben noch nicht diese Aufmerksamkeit

1 Zur Energiesicherheit Deutschlands und Europas, die hier nicht Gegenstand der Analyse sein kann, s. u.a. Heinrich Kreft, Die geopolitische Dimension der Energiesicherheit aus deutscher und europäischer Sicht, in: Reinhard C. Meier-Walser (Hrsg.), Energieversorgung als sicherheitspolitische Herausforderung, München, 2007, S. 31-50. 